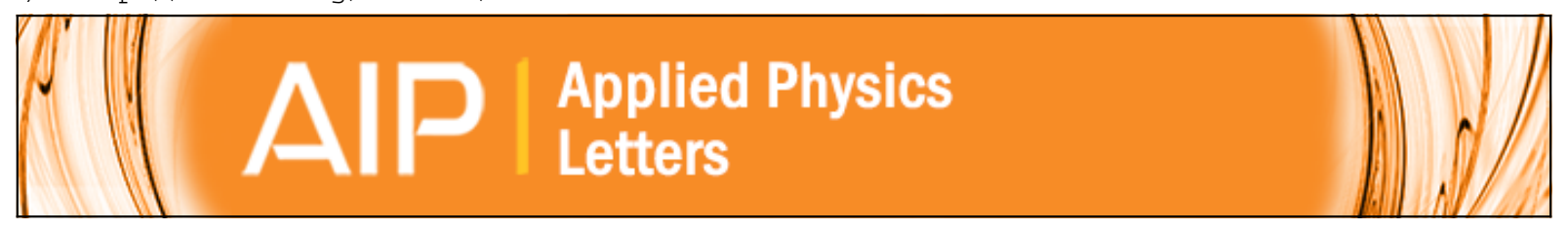

Near-ideal magnetoelectricity in high-permeability magnetostrictive/piezofiber laminates with a (2-1) connectivity

Shuxiang Dong, Junyi Zhai, Jiefang Li, and D. Viehland

Citation: Applied Physics Letters 89, 252904 (2006); doi: 10.1063/1.2420772

View online: http://dx.doi.org/10.1063/1.2420772

View Table of Contents: http://scitation.aip.org/content/aip/journal/apl/89/25?ver=pdfcov

Published by the AIP Publishing 


\title{
Near-ideal magnetoelectricity in high-permeability magnetostrictive/ piezofiber laminates with a (2-1) connectivity
}

\author{
Shuxiang Dong, ${ }^{\text {a) }}$ Junyi Zhai, Jiefang Li, and D. Viehland \\ Materials Science and Engineering, Virginia Tech, Blacksburg, Virginia 24060
}

(Received 2 October 2006; accepted 14 November 2006; published online 20 December 2006)

\begin{abstract}
Theoretically, the two-phase laminated configurations should have even much higher magnetoelectric (ME) effects-however, prior experimental studies have failed to find such an enhancement. Here, the authors report the unleashing of the potential of the (2-1) connectivity configuration: a piezofiber (one-dimension connectivity) layer laminated between two high-permeability magnetostrictive $\mathrm{FeBSiC}$ alloy ones (two-dimension connectivity) has near-ideal ME coupling. Very high ME effects of up to $22 \mathrm{~V} / \mathrm{cm} \mathrm{Oe}\left(4 \times 10^{-7} \mathrm{~s} / \mathrm{m}\right)$ at $1 \mathrm{~Hz}$-an order of magnitude higher than the giant ones-have been found. () 2006 American Institute of Physics.

[DOI: 10.1063/1.2420772]
\end{abstract}

Magnetoelectricity is the interaction between the polarization and magnetization subsystems of a solid. Early room temperature magnetoelectric (ME) measurements of $\mathrm{Cr}_{2} \mathrm{O}_{3}$ crystals $^{1,2}$ reported a value for the ME susceptibility of $\alpha_{\mathrm{ME}}=2.67 \times 10^{-12} \mathrm{~s} / \mathrm{m}$, or equivalently a ME field coefficient of $\alpha_{\mathrm{ME}}=\delta E / \delta H=0.01 \mathrm{~V} / \mathrm{cm} \mathrm{Oe}$, which remains to this day the highest value for a single phase material. Later, twophase magnetostrictive/piezoelectric particulate composites in (0-3), (1-3), and (3-3) connectivity 3,4 and laminated composites in a (2-2) connectivity ${ }^{5-14}$ were shown to have much higher ME effects. In particular, nonresonance giant $\mathrm{ME}$ field coefficients of up to $\alpha_{\mathrm{ME}}=2 \mathrm{~V} / \mathrm{cm} \mathrm{Oe}$ (or $\alpha_{\mathrm{me}} \approx 5$ $\times 10^{-8} \mathrm{~s} / \mathrm{m}$ or $\mathrm{C} / \mathrm{m}^{2} \mathrm{Oe}$ ) have been reported for (2-2) $(L-T)$ (longitudinally magnetized transversely poled) composites of piezoelectric $\mathrm{Pb}(\mathrm{Zr}, \mathrm{Ti}) \mathrm{O}_{3}$ (PZT) layers laminated with magnetostrictive $\mathrm{Tb}_{1-x} \mathrm{Dy}_{x} \mathrm{Fe}_{2-y}$ or Terfenol-D, ${ }^{5}$ Permendur, ${ }^{6} \mathrm{Fe}-\mathrm{Ga},{ }^{7}$ or $\mathrm{NiFe}_{2} \mathrm{O}_{4}$ (Ref. 8) ones, which are orders of magnitude higher than those of single phase systems. Such giant values of $\alpha_{\mathrm{ME}}$ occur when the magnetostrictive layer are in a dc biased piezomagnetic state near $H_{\mathrm{dc}}$ $\approx 400-500$ Oe. In said laminates, the ME effect is a product tensor property - and not intrinsic to individual layerscombining the magnetoelastic and elastoelectric effects of individual layers, via an elastic coupling between layers: an applied $H$ produces an elastic strain in the magnetostrictive phase that is stress coupled to that of the piezoelectric one, resulting in an induced voltage.

Theoretical investigations ${ }^{9,10}$ have shown that two-phase laminates in the $(L-L)$ configuration should have yet significantly higher values of $\alpha_{\mathrm{ME}}$ - one to two orders of magnitude - than that which has been experimentally reported. ${ }^{10}$ In fact, theoretically, the (L-L) mode should have by far the highest values of $\alpha_{\mathrm{ME}}$ than any other. The theoretical limit of $\alpha_{\mathrm{ME}}^{(L-L)}$ (in V/cm Oe) in the case of perfect or ideal ME coupling is given as

$$
\alpha_{\mathrm{ME}}^{(L-L)}=\frac{n d_{33, m} g_{33, p}}{n s_{33}^{E}\left(1-k_{33}^{2}\right)+(1-n) s_{33}^{H}} \quad(0<n<1),
$$

where $s_{33}^{E}$ and $s_{33}^{H}$ are the elastic compliances for the piezoelectric and magnetostrictive layers, $k_{33}$ the electromechanical coupling coefficient of the piezoelectric layer, $d_{33, m}$ and $g_{33, p}$ the longitudinal piezomagnetic- and piezoelectric-

${ }^{a)}$ Electronic mail: sdong@vt.edu voltage coefficients, and $n$ a thickness fraction of magnetostrictive layers. Equation (1) neglects (i) the demagnetization factor's effect due to our long-type construction and (ii) the direct effect of the magnetic permeability $(\mu)$ which is indirectly accounted for by the effective piezomagnetic coefficient: $d_{33, m}=\mu_{33}^{S} s_{33}^{H} \lambda_{33}$, where $\lambda_{33}$ is the magnetostriction coefficient. Equation (1) predicts a large $\alpha_{\mathrm{ME}}$ of $\sim 10 \mathrm{~V} / \mathrm{cm}$ Oe for a $(L-L)$ Terfenol-D/PZT ME laminate (for $n=0.5$ ), which is in significant discrepancy with the experimentally observed value of $\alpha_{\mathrm{ME}}=0.5 \mathrm{~V} / \mathrm{cm} \mathrm{Oe}$ [in our previous reports, ${ }^{10}$ a rectifying factor $\beta$ (in $N / V$ ) was empirically introduced for Eq. (1) adjustment]. Clearly, in spite of the significant progress made in the discovery of giant $\mathrm{ME}$ effects, ${ }^{5-12}$ only a fraction of the potentially available magnetoelectricity has been released for the $(L-L)$ configuration.

However, in prior reports, several important things had not been thoroughly considered that have significant ramifications on the encroachment of $\alpha_{\mathrm{ME}}^{(L-L)}$ upon this theoretical limit. First, the giant magnetostriction in ferromagnetic materials (Terfenol-D, Fe-Ga, etc.) used in prior studies had quite low relative magnetic permeabilities: $\mu_{r}<10$. This results in correspondingly low effective piezomagnetic coefficients and larger required magnetic biases: it is $d_{33, m}$ (i.e., $d \lambda / d H)$ and not magnetostriction $\lambda$ that matters. This fact,

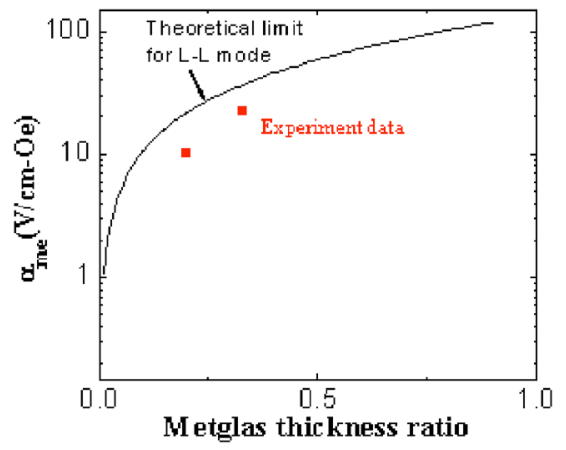

FIG. 1. (Color online) Graph of theoretical limitation of $\alpha_{\mathrm{ME}}^{(L-L)}$ as a function of thickness fraction $(n)$ of the magnetostrictive layers, calculated using Eq. (1) and shown as a solid line. Experimental data points are also shown in this investigation using piezofiber/Metglas $(L-L)$ laminates. Note that unlike the $L-T$ mode, when $n \rightarrow 1$ for the $L-L$ construction, $\alpha_{\mathrm{ME}}^{(L-L)}$ does not tend to zero; rather, it tends to a maximum. This is because for the $L-L$ mode, the polarization is along the length (or longitudinal axis) of the laminate, and thus with decreasing layer thickness the force imposed by a magnetostrictive layer of constant thickness increases. 


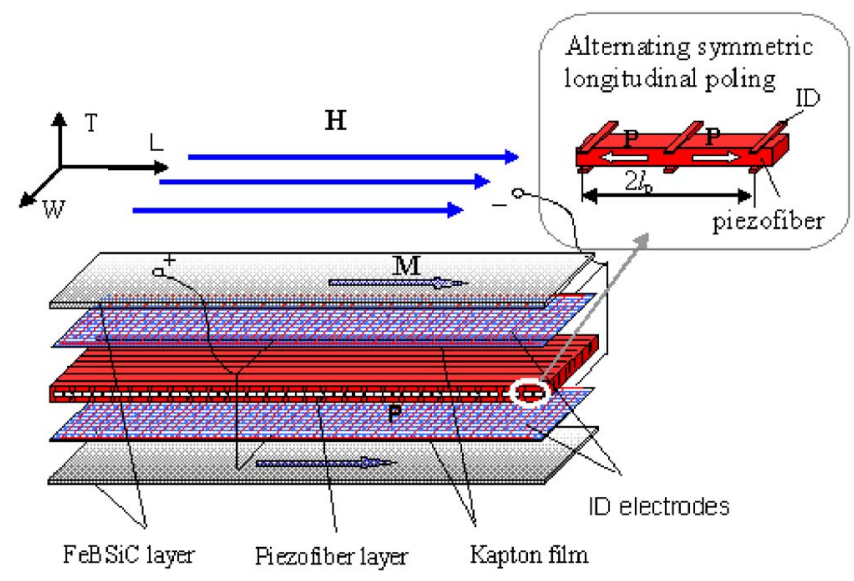

FIG. 2. (Color online) Illustration of our FeBSiC/piezofiber $(L-L)$ laminate configuration. It consists of a $1 \mathrm{D}$ piezoelectric active fiber/epoxy composited (AFC) thin layer where the fibers are oriented along the longitudinal axis, which is laminated between two $2 \mathrm{D}$ FeBSiC layers. Insulating Kapton films with interdigitated (ID) electrodes were placed between layers. Each piezofiber had numerous alternating symmetric longitudinally poled "pushpull" units) that were each $2 l_{p}=1 \mathrm{~mm}$ in length, as shown in the inset.

even though previously well known as given by Eq. (1), was not fully appreciated. Second, and more importantly, following Eq. (1), $\alpha_{\mathrm{ME}}^{(L-L)}$ is proportional to the piezoelectric-voltage $g_{33, p}$ coefficient, rather than piezoelectric constant: $d_{33, p}$ $=g_{33, p} \varepsilon_{33}$, where $\varepsilon_{33}$ is the permittivity. This fact favors lamination of piezofibers [one-dimension (1D) phase connectivity] in a composite layer, rather than a monolithic piezoelectric one, to magnetostrictive layers. Early investigations of (1-3) piezocomposites were performed by Newnham et al. ${ }^{13}$ Recently, investigations of (1-3) ME composites consisting of PZT rods in a Terfenol-D matrix were reported by Shi et al. ${ }^{14}$ but unfortunately $\alpha_{\mathrm{ME}}$ was $<0.5 \mathrm{~V} / \mathrm{cm}$ Oe under a required $H_{\mathrm{dc}}$ of $2000 \mathrm{Oe}$.

Recently, ${ }^{13}$ we reported that high-permeability FeBSiC alloy exhibits large piezomagnetic coefficients $d_{33, m}$ at quite low dc magnetic biases and a large ME coefficient when bonded to a polyvinylidene fluoride piezopolymer layer. Here, we report a $(L-L)$ ME composite consisting of a 1D phase connected piezoelectric PZT-fiber layer laminated between two two-dimension (2D) phase connected highpermeability magnetostrictive $\mathrm{FeBSiC}$ alloy (Metglas) foils, forming a magnetoelectric laminate with a (2-1) phase connectivity. This laminate has experimental values of $\alpha_{\mathrm{ME}}^{(L-L)}$ approaching that of the theoretical limit calculated using Eq. (1), as shown in Fig. 1 by solid red points. Our findings demonstrate the unleashing of near-ideal magnetoelectricity latent in the $(L-L)$ configuration.

Figure 2 illustrates our new $(L-L)$ configuration, which consists of a piezofiber layer laminated between two highpermeability $\mathrm{FeBSiC}$ alloy ones. The $\mathrm{FeBSiC}$ layers were obtained from Metglas Inc. (Conway, SC), were foils of $25 \mu \mathrm{m}$ thickness, had a relative permeability of $\mu_{r}$ $>40000$ due to a low magnetocrystalline anisotropy, and had a low saturation magnetostriction of $\lambda_{s} \approx 40 \mathrm{ppm}$ at $H_{\text {dc }}<10$ Oe. The use of a thin-foil form offers the additional advantage of reducing eddy current losses at high frequencies. The piezo-fibers were PZT-5A ceramic ones that were $100 \mu \mathrm{m}$ in thickness, $350 \mu \mathrm{m}$ in width, and $30 \mathrm{~mm}$ in length. A (1-3) piezoelectric active fiber/epoxy composite (AFC) thin layer was then made, following a construction used in actuators. The piezofibers were oriented along the

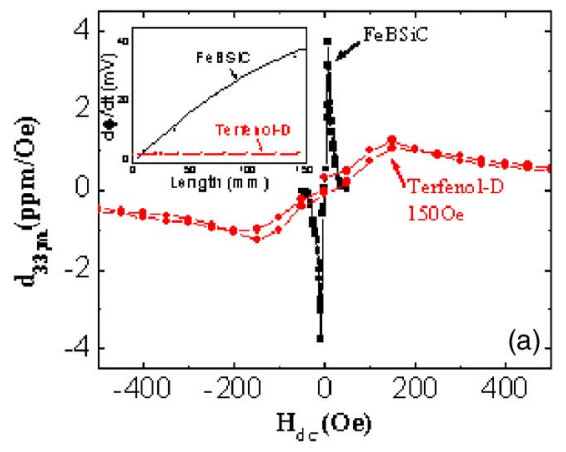

FIG. 3. (Color online) Effective piezomagnetic coefficients as a function of dc magnetic bias $H_{\mathrm{dc}}$ for ferromagnetic FeBSiC and Terfenol-D alloys. The inset shows the magnetic flux $(\delta \phi / \delta t)$ as a function of the length of the ferromagnetic layer, for both single FeBSiC (cross-sectional area: $A=7$ $\left.\times 0.025 \mathrm{~mm}^{2}\right)$ and Terfenol-D $\left(A=6 \times 1.5 \mathrm{~mm}^{2}\right)$ layers.

longitudinal axis of the laminate. Thin polymer (Kapton) insulating films with interdigititated (ID) electrodes were placed between PZT-fiber and FeBSiC layers, which were then assembled into a laminated composite using an epoxy resin. Each piezofiber had numerous alternating symmetric longitudinally poled "push-pull" units with a length of $2 l_{p}$ $=1 \mathrm{~mm}$, as shown in the inset of Fig. 2. This multiple pushpull $(L-L)$ configuration not only optimizes stress transfer, ${ }^{17,18}$ but also enhances the dielectric capacitance of the laminate. A second longer laminate was also constructed by laminating a (1-3) piezofiber layer (30 $\mathrm{mm}$ in length) between two long FeBSiC layers (100 mm in length). Following the composite nomenclature of Newnham et al. ${ }^{13}$ these ME laminates have a (2-1) connectivity of magneticpiezoelectric phases.

In Fig. 3, we show the effective $d_{33, m}$ as a function of $H_{\mathrm{dc}}$ for ferromagnetic FeBSiC and Terfenol-D alloy layers. These values were calculated from the slope of strictionmagnetic field $(\varepsilon-H)$ curves measured using a strain-gauge method. It can be seen that the maximum value of $d_{33, m}$ is $\sim 4 \mathrm{ppm} /$ Oe under $H_{\mathrm{dc}} \approx 10$ Oe for Metglas; whereas that of Terfenol-D was $\sim 1 \mathrm{ppm} /$ Oe under $H_{\mathrm{dc}} \approx 200$ Oe. Although Terfenol-D has much higher magnetostrictions $\lambda$, its effective $d_{33, m}$ is notably lower than that of a FeBSiC alloy. This difference reflects the much higher magnetic permeability of FeBSiC: a high $\mu_{r}$ results in a low saturation field, and thus a large value of $d \lambda / d H$ at low $H_{\mathrm{dc}}$. In addition, high- $\mu$ FeB$\mathrm{SiC}$ layers also concentrate magnetic flux $(d \phi / d t)$. The inset of Fig. 3 shows $d \phi / d t$ as a function of the length $(l)$ of FeBSiC and Terfenol-D layers, measured using a search coil. We found that the flux in FeBSiC layer (cross-section area is $\left.A=7 \times 0.025 \mathrm{~mm}^{2}\right)$ is much higher ( 20 times) than that of Terfenol-D $\left(A=6 \times 1.5 \mathrm{~mm}^{2}\right)$, and also that $d \phi / d t \sim l$ for the FeBSiC layer. These results demonstrate the potential of $\mathrm{FeBSiC}$ as a superior dc-biased piezomagnetic layer for ME laminate composites, one that can also operate under much reduced $H_{\mathrm{dc}}$.

We then measured the voltage induced across the ID electrodes of the piezofiber layer as a function of (i) $H_{\mathrm{dc}}$ in response to a constant ac $(f=1 \mathrm{kHz})$ magnetic drive of $H_{\mathrm{ac}}$ $=1 \mathrm{Oe}$, both applied along the length of the laminate, and (ii) $f$ under constant $H_{\mathrm{ac}}=1$ Oe and $H_{\mathrm{dc}}=4 \mathrm{Oe}$. An electromagnet was used to apply $H_{\mathrm{dc}}$, a pair of Helmholtz coils was used to generate a small $H_{\mathrm{ac}}$, and the induced voltage was measured by a lock-in amplifier. First, in Fig. 4(a), we show $\alpha_{\mathrm{ME}}^{(L-L)}$ as a function of $H_{\mathrm{dc}}$. Data are given for both short $(l=30 \mathrm{~mm})$ 

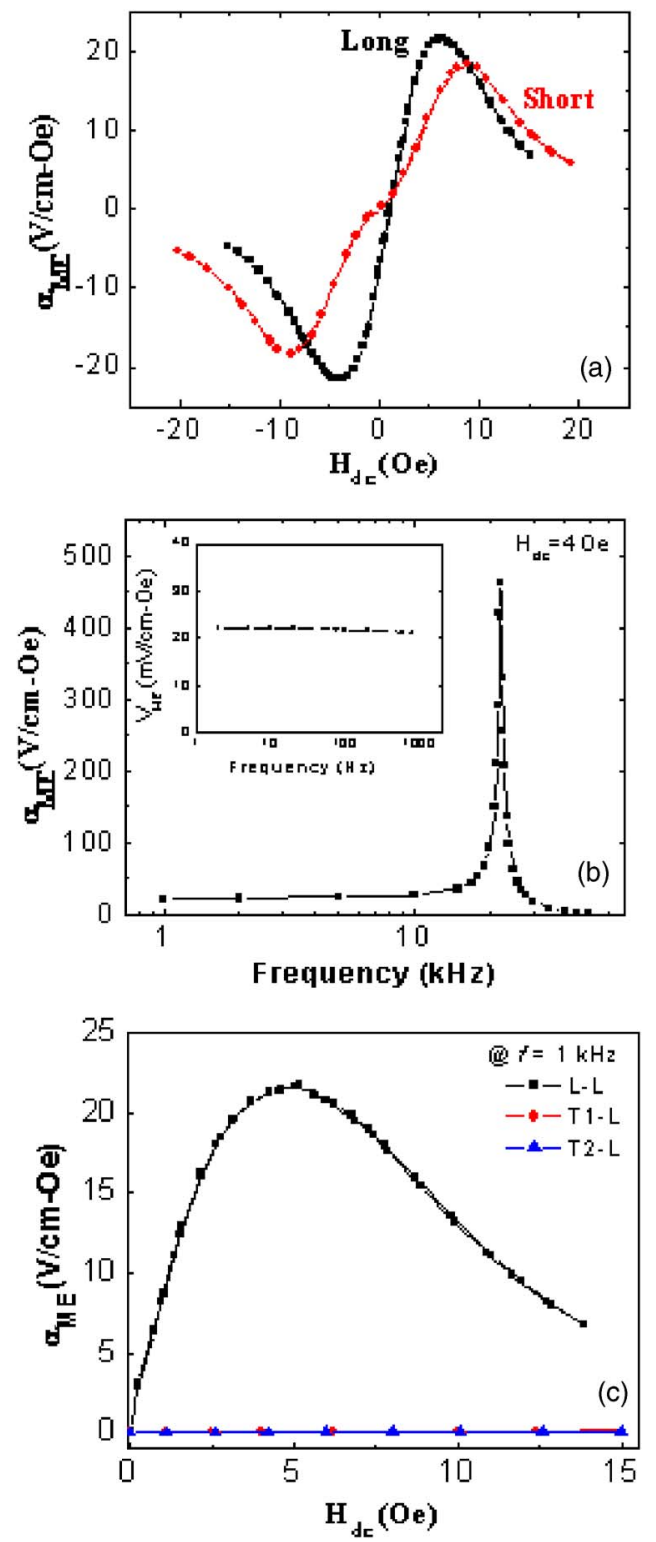

FIG. 4. (Color online) Magnetoelectric characterizations of $\mathrm{FeBSiC} /$ piezofiber laminates: (a) the $(L-L) \mathrm{ME}$ voltage coefficient $\alpha_{\mathrm{ME}}^{(L-L)}$ as a function of $H_{\mathrm{dc}}$ for both short $(30 \mathrm{~mm})$ and long $(100 \mathrm{~mm})$ laminates; (b) $\alpha_{\mathrm{ME}}^{(L-L)}$ as a function of frequency illustrating a strong enhancement at the electromechanical resonance frequency, where the inset shows a flat response over the quasistatic frequency range; and (c) anisotropy of $\alpha_{\mathrm{ME}}$ for $H_{\mathrm{ac}}$ applied along the length $\left(\alpha_{\mathrm{ME}}^{(L-L)}\right)$, width $\left(\alpha_{\mathrm{ME}}^{(T 1-L)}\right)$, and thickness $\left(\alpha_{\mathrm{ME}}^{(T 2-L)}\right)$ of the laminate.

and long $(l=100 \mathrm{~mm})$ laminates, demonstrating that (i) ME coupling in $\mathrm{FeBSiC} /$ piezofiber laminates is much higher than that in Terfenol-D/monolithic-piezoelectric laminates; and (ii) $\alpha_{\mathrm{ME}}^{(L-L)} \sim l$ due to magnetic flux concentration effect, as shown in Fig. 3, which results in stronger magnetic induction in ME laminates. These data reveal maximum $\alpha_{\mathrm{ME}}^{(L-L)}$ values of 18 (short) and $22 \mathrm{~V} / \mathrm{cm}$ Oe (long) under $H_{\mathrm{dc}}$ of 8 and 4 Oe, respectively: correspondingly $\alpha_{\mathrm{ME}}^{(L-L)}=3.2 \times 10^{-7}$ and $4 \times 10^{-7} \mathrm{~s} / \mathrm{m}$ or $\mathrm{C} / \mathrm{m}^{2}$ Oe. These maximum values are much higher than recently reported giant ones $\left(\alpha_{\mathrm{ME}}^{(L-T)}\right.$ $\sim 0.5-2.2 \mathrm{~V} / \mathrm{cm} \mathrm{Oe}^{5-8}{ }^{5-8} \quad \alpha_{\mathrm{ME}}^{(L-L)} \sim 0.5 \mathrm{~V} / \mathrm{cm} \mathrm{Oe} ;{ }^{10,11} \quad H_{\mathrm{dc}}$ $=300 \mathrm{Oe}$ ) and require much lower biases ( $<60$ times). These values of $\alpha_{\mathrm{ME}}^{(L-L)}$ approach the theoretical limiting ones for the case of near-ideal ME coupling given by Eq. (1) (with $n$ $=0.33$ ), as summiarized in Fig. 1 . These very high effects occur due to (i) optimum stress transfer in the $(L-L)(2-1)$ configuration, (ii) the large $d_{33, m}$ and high permeability of the FeBSiC alloy layer, and (iii) the high $g_{33, p}$ of the 1D piezofiber layer. Theoretically, further enhancements in ME coupling are possible by increasing the magnetic phase ratio $n$ (see Fig. 1).

Next, in Fig. 4(b), we show $\alpha_{M E}^{(L-L)}(l=100 \mathrm{~mm})$ as a function of frequency under a constant bias of $H_{\mathrm{dc}}=4 \mathrm{Oe}$. The inset demonstrates that very high values are maintained down to quasistatic frequencies. The figure itself reveals a strong resonance enhancement of $\alpha_{M E}^{(L-L)}$ to $\sim 500 \mathrm{~V} / \mathrm{cm}$ Oe at $f_{r} \approx 22 \mathrm{kHz}$ or, correspondingly, $\alpha_{\mathrm{ME}}^{\left(-L^{M}\right)}=10^{-5} \mathrm{~s} / \mathrm{m}$. Finally, in Fig. 4(c), we show $\alpha_{\mathrm{ME}}-H_{\mathrm{dc}}$ for $H_{\mathrm{ac}}$ applied along the length $\left(\alpha_{\mathrm{ME}}^{(L-L)}\right)$, width $\left(\alpha_{\mathrm{ME}}^{(T 1-L)}\right)$, and thickness $\left(\alpha_{\mathrm{ME}}^{(T 2-L)}\right)$ of the laminate. The data show that $\alpha_{\mathrm{ME}}^{(L-L)}$ is dramatically larger than either $\alpha_{\mathrm{ME}}^{(T 1-L)}$ or $\alpha_{\mathrm{ME}}^{(T 2-L)}$, with a large anisotropy factor of $100<K=\alpha_{\mathrm{ME}}^{(L-L)} / \alpha_{\mathrm{ME}}^{(T-L)}<1000$, which can be attributed to the unidirectional natures of the (i) length-strain sensitivity of $1 \mathrm{D}$ piezofibers and (ii) demagnetization factor $N$ of Metglas ribbon.

In summary, we have unleashed near-ideal magnetoelectricity latent in (2-1) laminates, consisting of a 1D connected piezoelectric PZT-fiber layer laminated between two 2D connected high-permeability magnetostrictive FeBSiC foils. Specifically, we find very high ME coefficients of $\alpha_{\mathrm{ME}}^{(L-L)}$ $>20 \mathrm{~V} / \mathrm{cm}$ Oe under small biases of $5 \mathrm{Oe}$, approaching predicted theoretical limits; (i) that are frequency independent in the quasistatic range; (ii) whose values are strongly enhanced, $\alpha_{\mathrm{ME}}^{(L-L)} \sim 500 \mathrm{~V} / \mathrm{cm} \mathrm{Oe}$, at the resonance frequency; and (iii) whose response is highly anisotropic, offering unidirectional sensitivity.

This work was supported by the Office of Naval Research and DARPA.

${ }^{1}$ D. N. Astrov, Sov. Phys. JETP 13, 729 (1961).

${ }^{2}$ V. J. Folen, G. T. Rado, and E. W. Stalder, Phys. Rev. Lett. 6, 607 (1961). ${ }^{3}$ C.-W. Nan, Phys. Rev. B 50, 6082 (1994).

${ }^{4}$ G. Harshe, J. P. Dougherty, and R. E. Newnham, Int. J. Appl. Electromagn. Mater. 4, 161 (1993).

${ }^{5}$ S. X. Dong, J. F. Li, and D. Viehland, Appl. Phys. Lett. 83, 2265 (2003); J. Appl. Phys. 95, 2625 (2004).

${ }^{6}$ U. Lalestin, N. Padubnaya, G. Srinivasan, and C. P. Devreugd, Appl. Phys. A: Mater. Sci. Process. 78, 33 (2004).

${ }^{7}$ S. X. Dong, J. Zhai, F. Bai, J. Li, D. Viehland, and T. Lograsso, J. Appl. Phys. 97, 103902 (2005).

${ }^{8}$ G. Srinivasan, E. Rasmussen, J. Gallegos, R. Srinivasan, Y. Bokhan, and V. Laletin, Phys. Rev. B 64, 214408 (2001); G. Srinivasan, E. T. Rasmussen, and R. Hayes, ibid. 67, 014418 (2003).

${ }^{9}$ M. Bichurin, V. Petrov, and G. Srinivasan, Phys. Rev. B 68, 054402 (2003).

${ }^{10}$ S. X. Dong, J. F. Li, and D. Viehland, IEEE Trans. Ultrason. Ferroelectr. Freq. Control 50, 1253 (2003); 51, 794 (2004); Appl. Phys. Lett. 85, 5305 (2004).

${ }^{11}$ S. X. Dong, J. F. Li, and D. Viehland, Appl. Phys. Lett. 85, 2307 (2004).

${ }^{12}$ J. Ryu, A. Vazquez Carazo, K. Uchino, and H. Kim, Jpn. J. Appl. Phys., Part 1 40, 4948 (2001).

${ }^{13}$ R. E. Newnham, D. P. Skinner, and L. E. Cross, Mater. Res. Bull. 13, 525 (1978).

${ }^{14}$ Z. Shi, C. W. Nan, J. Zhang, N. Cai, and J.-F. Li, Appl. Phys. Lett. 87, 012503 (2005).

${ }^{15}$ J. Y. Zhai, S. X. Dong, J. F. Li, and D. Viehland, Appl. Phys. Lett. 89, 083507 (2006).

${ }^{16}$ A. A. Bent and N. W. Hagood, J. Intell. Mater. Syst. Struct. 8, 903 (1997).

${ }^{17}$ S. X. Dong, J. Y. Zhai, F. M. Bai, J. F. Li, and D. Viehland, Appl. Phys. Lett. 87, 062502 (2005).

${ }^{18}$ C. R. Bowen, R. Stevens, L. J. Nelson, A. C. Dent, G. Dolman, B. Su, T. W. Button, M. G. Cain, and M. Stewart, Smart Mater. Struct. 15, 295 (2006). terms at: hitp://scitation.aip.org/termsconditions. Downloaded to IP: 